\title{
Trust-less electricity consumption optimization in local energy communities
}

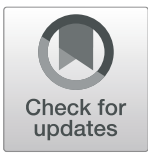

\author{
Fabian Knirsch*, Oliver Langthaler and Dominik Engel \\ From The 8th DACH+ Conference on Energy Informatics, \\ Salzburg, Austria. 26-27 September, 2019
}

\author{
${ }^{*}$ Correspondence: \\ fabian.knirsch@en-trust.at \\ Center for Secure Energy \\ Informatics, Salzburg University of \\ Applied Sciences, Urstein Süd 1 \\ 5421, Puch b. Hallein, Austria
}

\begin{abstract}
Optimizing energy consumption in local energy communities is one of the key contributions to the so-called smart grid. Such communities are equipped with rooftop photovoltaic power plants or other forms of small power plants for local energy production. In addition, a number of appliances allow for shiftable energy consumption, e.g., heat pumps or electric vehicle charging stations. The ability to shift is, however, dependent on customer preferences. In this paper, we present a trust-less approach for optimizing the electricity consumption in a local energy community given forecasts of energy production and customer demands, along with constraints for shiftable loads. In larger communities, appointing a single party for managing load curtailment requires a high level of trust. In the proposed trust-less approach, all parties can independently propose optimal solutions for this optimization problem and then globally agree one one solution that meets the defined requirements to the greatest extent.
\end{abstract}

Keywords: Local energy communities, Optimization, Directed acyclic graph

\section{Introduction}

Local energy communities (LECs) are defined as a number of residential households, industrial facilities and small power plants that aim at balancing energy demand and supply within a geographically bounded region (Van Der Schoor and Scholtens 2015). In order to minimize the need for larger wide-spanning power transmission grids, produced energy should be consumed within the community right at the time of production. The same concept holds - on a small scale - for residential households equipped with photovoltaic power plants, where energy produced from these power plants is preferably consumed from appliances within the same household. Erol-Kantarci and Mouftah (2010). Load shifting (i.e., adapting the running time of appliances to better meet the supply of currently available energy) and usage of household batteries is intended to optimize self-consumption, see e.g., Babrowski et al. (2014); Caron and Kesidis (2010); Cao et al. (2012); Rotering and Ilic (2011); Zakeri and Syri (2015).

For the smart grid, a decentralized network of energy producers and consumers, such approaches for electricity optimization are crucial and integral to the concept of LECs. Electricity consumption optimization is designed to avoid costly long-term investments in electricity transportation networks and communication infrastructure and allows for

(c) The Author(s). 2019 Open Access This article is distributed under the terms of the Creative Commons Attribution 4.0 International License (http://creativecommons.org/licenses/by/4.0/), which permits unrestricted use, distribution, and reproduction in any medium, provided you give appropriate credit to the original author(s) and the source, provide a link to the Creative Commons license, and indicate if changes were made. 
the use of locally produced energy from small power plants and often renewable resources (Khan and Khan 2013).

For curtailing load and optimizing electricity consumption for a mostly self-contained local energy community, appliances adapt their demand to the available electricity by shifting the load to times where, e.g., energy from photovoltaic power plants is available. While batteries may act as buffers and some appliances such as heat pumps or electric vehicles (EVs) allow for relatively easy load curtailment, other appliances are less flexible. Above all, customer requirements need to be taken into account.

This paper introduces a trust-less and decentralized approach for achieving consensus on a load curtailment plan. For a specified time period, all parties can announce electricity supply and demand with their respective constraints. All parties can then independently propose solutions for this optimization problem and globally agree on one solution that meets the defined requirements (i.e., maximization of the consumption of self-produced energy) to the greatest extent. The storage of the proposed solutions and each party's votes is based on a Directed Acyclic Graph (DAG).

The contribution of this paper is twofold: First, we present a trust-less approach for electricity consumption optimization. This approach does not need a central trusted party that is responsible for load curtailment and allows for a dynamic setting with changing participating devices, e.g., electric vehicles. Second, the proposed approach guarantees that a majority of the participants agree to the proposed load curtailment plan.

Note that the scope of this paper is primarily on the information layer, i.e., how to optimize and balance produced and consumed energy, but not on the physical layer or the market layer. Any forms of billing or energy trading can, however, be built on top of the proposed protocol, following concepts such as, e.g., Munsing et al. (2017); Sorin et al. (2019).

The rest of the paper is structured as follows: "Related Work" section provides an overview of state-of-the-art work in the field of energy consumption optimization. "Preliminaries" section introduces the formalization for load curtailment requests and optimizations, a notion of shiftable load and the concept of DAGs. "Optimization Protocol" section describes the proposed protocol to achieve consensus on a load curtailment plan. "Evaluation" section evaluates the proposed protocol with respect to scalability, malicious behavior as well as effectiveness and "Conclusion" section summarizes this paper and gives an outlook regarding future work.

\section{Related Work}

Related work in the field of energy consumption optimization can be roughly clustered in the following areas: (i) blockchain-based approaches that address the market layer and balance demand and supply via pricing; (ii) approaches that incorporate social networks for load balancing; (iii) peer-to-peer market approaches that focus on demand-side management; and (iv) battery-based approaches that aim at maximizing the consumption of self-produced energy.

Mengelkamp et al. focus on energy markets for microgrids, see, e.g., Mengelkamp et al. (2018a, b). Their work is mostly on balancing supply and demand on the market layer with a blockchain as a decentralized data structure. In our work, we focus on finding an optimal solution for load curtailment, rather than focusing on pricing. We further use a DAG, which is a more recent development and has some advantages over classical blockchains 
in terms of scalability and partition tolerance. In Munsing et al. (2017), blockchains are used for the decentralized optimization of energy resources in microgrids and in Sorin et al. (2019), a peer-to-peer market structure based on a multilateral economic dispatch formulation is introduced. In contrast to this paper, where a DAG is used for finding a solution the majority agrees on, their work uses smart contracts that automatically trigger payments. The latter is out of scope for this work, as we focus on finding consensus for load curtailment.

Social networks as a basis for the coordination of energy consumption in the form of virtual energy communities are explored in, e.g., Skopik (2014) and Huang et al. (2015). While the large-scale impact of social networks is an interesting aspect, this is out of the scope of our work.

An overview and a taxonomy of demand side management measures, along with an analysis of the various types of demand side management systems, is provided in Palensky and Dietrich (2011). In order to explore the potential of such systems, the structural design of a possible demand side management system for households is roughly outlined in Lei et al. (2012).

Approaches aiming to maximize the amount of self-consumed PV production within a household using battery storage can be found in, e.g., Weniger et al. (2014), Van der Kam and Van Sark (2014) and Martins et al. (2016). The approaches proposed in these papers can be used as a basis for the algorithm for finding the optimal solution. However, the work presented in this paper is specifically designed to work in heterogeneous, dynamic environments within a trust-less setting, rather than on a household level.

Lastly, Koirala et al. (2016) perform a review of key issues and trends shaping integrated community energy systems. Their work draws a bigger picture and the identified roles and responsibilities can serve as the basis for the future shaping of LECs.

\section{Preliminaries}

This section introduces the preliminaries for trust-less electricity consumption optimization.

\section{Participants}

Participants are persons or legal entities controlling one or more devices that consume and/or produce electrical energy. For instance, a participant may be a household including a PV plant, a water heater, a washer-dryer and a dishwasher. In an LEC, temporary visitors such as EV owners may also register as participants. Within the optimization protocol, each participant is uniquely identified via a public-private key pair (see e.g., Johnson et al. (2001)) generated during the registration process. The private key can then be used to sign transactions and to vote for solutions.

\section{Formalization}

In order to formalize algorithms that optimize the electricity consumption, a formal representation of predictions for production and consumption is needed. Such a formalization is presented in, e.g., Munsing et al. (2017); Sorin et al. (2019); Taneja and Culler (2010); Chetto (2014). However, these formalizations do not allow individual appliances or participants to formalize their forecast for demand and supply in a concise, selfcontained way that is suitable for decentralized optimization. For the proposed scheme, 
an approach that is tailored towards the specific needs of transaction-based processing is needed. A transaction is a prediction that includes capacities and preferences for curtailment scheduling.

Each request for energy consumption optimization $T_{k}$ is partitioned into a total of $N_{k}$ non-overlapping arbitrary length time slots $t_{k, 0}$ to $t_{k, N-1}$. For this paper, we assume such a period to be one day $(24 \mathrm{~h})$ and the smallest addressable time slot to be $15 \mathrm{~min}$. For example, one day can be divided into 15 min intervals $t_{k, 0}$ to $t_{k, 95}$ of equal length or in intervals of arbitrary length, e.g., $t_{k, 0}$ from 00:00 to 06:00, $t_{k, 1}$ from 06:15 to 20:00 and $t_{k, 2}$ from 20:15 to 23:45.

A transaction for a (shiftable) load $T$ (index $k$ is omitted for readability), which predicts the energy demand for the next day, is defined as follows:

$$
T=\left\{E^{\mathrm{tot}} ; t_{0}, \ldots, t_{N}\right\}, t_{i}=\left\{E^{\mathrm{min}}, E^{\mathrm{max}} ; t^{\text {earliest }}, t^{\text {latest }} ; \Delta\right\}
$$

where $E^{\text {tot }}$ is the total amount of energy needed between the total possible duration $t_{0}$ to $t_{N-1}$ and each of the $t_{i}, 0 \leq i \leq N-1$ represents a request for energy between $E^{\mathrm{min}}$ and $E^{\text {max }}$ for time slot $i$ with a duration of $\Delta$ given in numbers of 15 min intervals. $t^{\text {earliest }}$ and $t^{\text {latest }}$ defines the earliest beginning and the latest ending time for this time slot, which can also be set to $*$ for an arbitrary starting and ending time, respectively. In some cases, it might be necessary to define a time period spanning more than $24 \mathrm{~h}$. When a time refers to the next day, this is denoted by +1 , e.g., 06:00 +1 . In order to shift the load of an appliance which defined its demand or supply in a transaction $T$, all $t_{i}$ in $T$ are in a list and must occur in the specified order, i.e., for shifting load, $t_{i+1}$ must not occur before $t_{i}$. This does, however, not affect the time slots defined by other transactions, which can be scheduled independently. $e$ represents a demand for energy and thus uses positive real numbers, whereas a supply is represented by negative real numbers.

Table 1 provides a summary of the notation and symbols used in this paper.

Following this pattern, a non-shiftable, constant load is defined by

$$
T=\left\{e ; t_{0}\right\}, t_{0}=(e, e ; 00: 00,23: 45 ; 96) .
$$

\begin{tabular}{|c|c|}
\hline$\overline{T_{k}}$ & Transaction $k$ for demand request or supply forecast \\
\hline$E^{\text {tot }}$ & Total demand or supply of energy \\
\hline$t_{k, i}$ & Time slot $0 \leq i \leq N_{k}$ for demand or supply forecast \\
\hline$N_{k}$ & Total number of time slots in transaction $k$ \\
\hline$E^{\min }$ & Min. demand ( $E^{\min } \geq 0$ ) or supply $\left(E^{\min }<0\right.$ ) for time slot \\
\hline$E^{\max }$ & Max. demand $\left(E^{\min } \geq 0\right.$ ) or supply $\left(E^{\min }<0\right.$ ) for time slot \\
\hline$t_{\text {earliest }}$ & Earliest start time for time slot, $t_{i+1}^{\text {earliest }} \geq t_{i}^{\text {latest }}$ \\
\hline$t^{\text {latest }}$ & Latest end time for time slot, $t_{i-1}^{\text {latest }}<t_{i}^{\text {earliest }}$ \\
\hline$\Delta$ & Duration of demand or supply during time slot \\
\hline$\longleftrightarrow$ & Time slot of fixed length $\Delta=t^{\text {latest }}-t^{\text {earliest }}$ \\
\hline$\longleftrightarrow / \longleftrightarrow$ & Extended time slot of fixed length interrupted for display \\
\hline$\langle\ldots \ldots \ldots\rangle\rangle$ & Time slot of flexible length $\Delta \leq t^{\text {latest }}-t^{\text {earliest }}$ \\
\hline MIMIMV & Flexible demand or supply between $E^{\min }$ and $E^{\max }$ \\
\hline$\square$ & Fixed demand or supply $E^{\min }$ \\
\hline
\end{tabular}

Table 1 Notation and Symbols for Transactions 
The total amount of energy $E^{\text {tot }}=e$, where $e$ is both the minimum and maximum energy (thus constant) for a single time slot spanning the entire day (covering 96 intervals of 15 $\min )$.

Additionally, transactions need a global ordering, i.e., the time at which the request first occurred relatively to all other requests. This is denoted by an index $k$ for each transaction $T$.

\section{Solutions}

A solution $S_{i} \in S$ is a proposal for load curtailment that incorporates all of the transactions for the current optimization period. The set of all solutions proposed at a given point in time is denoted as $S$. In order to allow participants to agree on a solution, a global set of criteria needs to be defined. Note that, while the criteria are public, the used optimization algorithm can be chosen by each participant independently.

An optimal solution $S_{\text {opt }}$ meets the following requirements, given the set of all transactions $T_{\text {prod }}$ (i.e., the set of transactions representing the energy produced) and $T_{\text {cons }}$ (i.e., the set of transactions representing the energy consumed) that are currently available:

$$
S_{\text {opt }}=\underset{S_{k} \in S}{\operatorname{argmax}}\left\{\sum_{i=0 \ldots N} \min \left(\sum_{T_{p} \in T_{\text {prod }} \cap S_{k}}\left|e\left(t_{i, T_{p}}\right)\right|, \sum_{T_{c} \in T_{\text {cons } \cap S_{k}}}\left|e\left(t_{i, T_{c}}\right)\right|\right)\right\}
$$

Non-formally, this can be expressed as the requirement that the amount of self-produced energy that is consumed is maximal. In the set of transactions representing the energy produced that are part of the currently evaluated solution $S_{k}$, the energy supply for each time slot $t_{i}$ is summed up. Similarly, in the set of transactions representing the energy consumed that are part of the same solution $S_{k}$, the energy consumption for each time slot $t_{j}$ is summed up. The lower value corresponds to the amount of self-produced energy that is also self-consumed.

Additionally, the following constraints must be met for a load curtailment solution:

1. An energy demand of $E^{\text {tot }}$ for each transaction and for the full period must be provided.

2. For each time slot, the energy must not be less than $E^{\text {min }}$ or more than $E^{\text {max }}$.

3. All $t_{i}$ must occur in the defined order.

4. Time slots of fixed length must occur in exactly this length.

5. Time slots of variable length must occur within the defined interval.

\section{Shiftable Loads}

In typical modern and future households, there is a number of loads which can be expected to be shiftable to some extent. Examples include EV or household battery charging, dish and laundry washing as well as HVAC, heat pump and storage water heater duty cycles. The extent to which a given load is shiftable may depend on a variety of factors such as user preferences and the sizing (and thus the performance reserves) of the system. Furthermore, some loads such as EV charging may be variable both in time and power usage, while others such as dish washers may only be variable in starting time.

The following shows sample transactions for an EV battery and a PV plant. A formal representation of their demand and supply, respectively, as a transaction, can be found in the "Appendix" section. Figure 1 shows a transaction for a household or EV battery, 


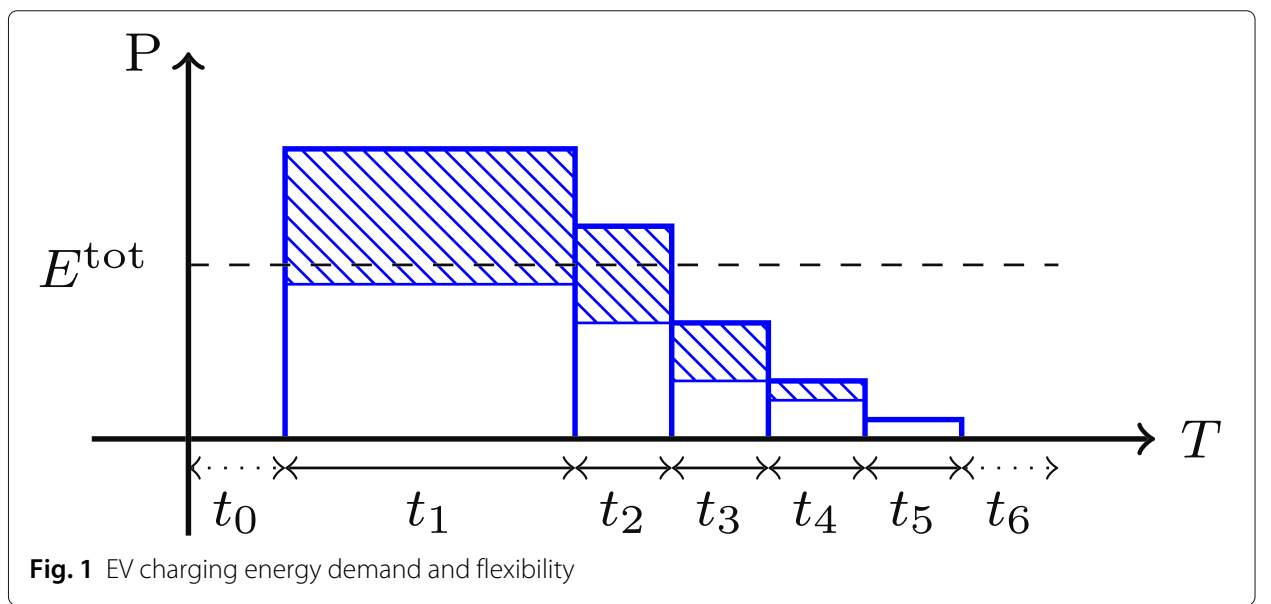

requesting energy over time, given the typical load curve of such a battery. While there is a large capacity for consuming more energy during $t_{1}$, the amount needed between $t_{2}$ and $t_{5}$ is decreasingly flexible. Figure 2 is a visualization of a forecast for the production of a given PV plant from a residential household.

\section{Directed Acyclic Graphs}

For achieving consensus and for globally agreeing on an optimal load curtailment plan, a distributed, immutable and transparent storage for transactions is required. Such a distributed storage allows to achieve consensus without the need for a central trusted party, which makes the overall approach trust-less.

It has been shown in Karlsson et al. (2018), that a DAG provides the necessary properties for achieving a decentralized common state and thus represents an ordered set of dependent transactions. DAGs have similar features as blockchains (see, e.g., Nakamoto (2008); Tschorsch and Scheuermann (2016)). While blockchains order transactions and bundle them in blocks where the global consistent state is represented by the longest branch, DAGs represent this state by having a majority of votes on certain transactions from the participants (Benčić and Žarko 2018). Votes are represented by digital signatures of participants that incorporate a number of existing and valid transactions into a new one. DAGs are also more partition-tolerant and come at increased scalability (Karaarslan and Adiguzel 2018).

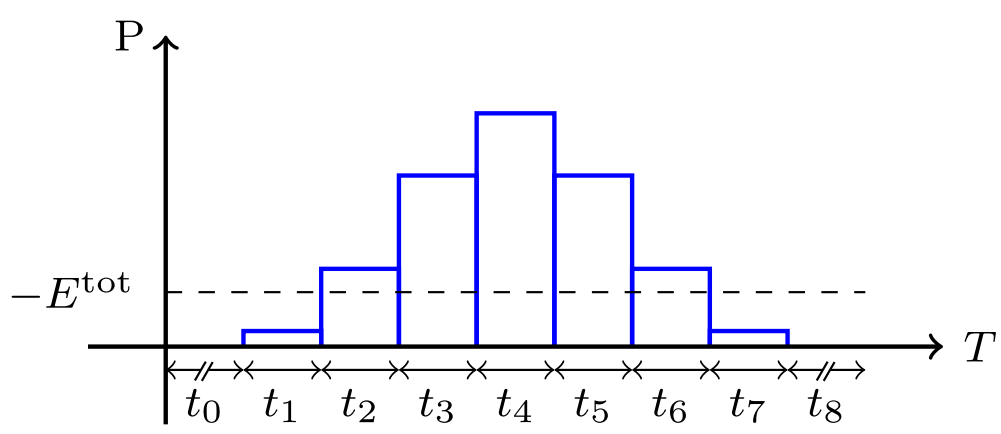

Fig. 2 PV production energy supply 
It has to be noted that, for the setup needed in the proposed protocol, the DAG is operated in a permissioned environment, i.e., all participants are known to each other and register themselves to some authority. In practice, this authority can be the local DSO. However, this authority is not involved in achieving consensus on optimal consumption. It merely serves as the hub for managing participants and access rights.

For achieving a distributed consensus, majority voting is used. All participants share the same information and accept a certain transaction or not, depending on whether it meets the previously agreed-upon requirements. In practice, this voting is reflected by digitally signing a transaction with a participant's private key, which uniquely identifies a participant.

In summary, the following properties of DAGs are relevant for the proposed application:

- Authenticity. All transactions are authenticated and the participant who committed the data to the DAG is uniquely identified, e.g., by a public key or an ID.

- Immutability. Once transactions are written to the DAG and other users have agreed on the validity of that data, it is linked to all following transactions by a cryptographic hash function and thus is not mutable without rebuilding significant portions of the entire data structure.

- Contribution. All participants must actively work on checking the validity of data when wanting to submit own transactions. In particular, new transactions must confirm previous valid transactions by linking them via their cryptographic hash value.

- Consensus. All participants vote on the validity of transactions and thus agree on a global state. Valid transactions are confirmed, whereas invalid transactions are not further processed.

The following section explains how these features of the DAG are used to set up the optimization protocol.

\section{Optimization Protocol}

All participants are connected in a peer-to-peer network and use a DAG as a decentralized common data structure for maintaining a globally consistent state. Figure 3 provides an overview of the setup and how the participants are connected. All participants, e.g., households, appliances or EVs, register at the local DSO and then commit their electricity consumption and production or a solution as a transaction to the DAG.

Figure 4 shows an exemplified run of the optimization protocol given a number of such transactions. Two participants submit a number of transactions $T_{0}$ to $T_{3}$ and propose corresponding solutions $S_{0}$ to $S_{3}$. New transactions may only be added alongside according solutions, which ensures that at any point in time, there exists an agreed upon solution for all submitted valid transactions. Each solution incorporates all previous valid transactions and equals or improves upon the previous valid solution with regard to the requirement defined in Eq. 1. Note that this requirement does not constitute a dilemma for the sender of a new transaction, because the most trivial solution may simply add the new transaction on top of the previous solution and thereby does not incur any computationally intensive operations. Subsequently, superior solutions (depicted by $S_{3}$ in Fig. 4) may be found 


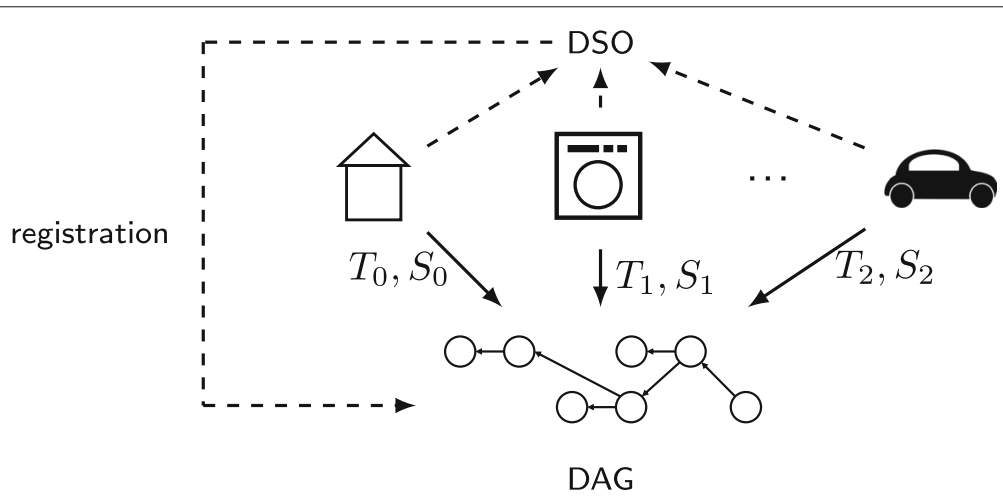

Fig. 3 Overview of the proposed protocol and the communication links. Participants (e.g., households, appliances and EVs) register at the DSO and then commit their load forecasts to the DAG

and added within the optimization period. In the following, each step of the protocol is described in detail.

\section{Registration and Setup}

New participants (e.g., households or temporary users such as visiting EV owners) can join at any time by registering at the local DSO or LEC administrator to receive permission to send transactions. This registration is necessary for maintaining a list of participants for the following round of optimization and subsequently for accounting purposes. Note that the DSO and the LEC administrator are only required for administrative purposes and do not need to be trusted parties with regard to finding solutions.

\section{Demand And Supply Transaction}

Any participant can submit a transaction $T$ (as described in "Preliminaries" section) for demand or supply of energy at any time. These transactions are sent to all participants via a peer-to-peer network. Note that this a forecast and a best effort estimation, rather than a binding commitment to produce or consume a certain amount of energy.

\section{Solution Commitment}

Any participant can submit a solution $S$ for matching demand to supply and thus optimizing the electricity consumption. If a new transaction is sent, it must include an appropriate solution. These solutions are sent to all participants via a peer-to-peer network. The quality of the solution is assessed given the criteria established in "Preliminaries" section.

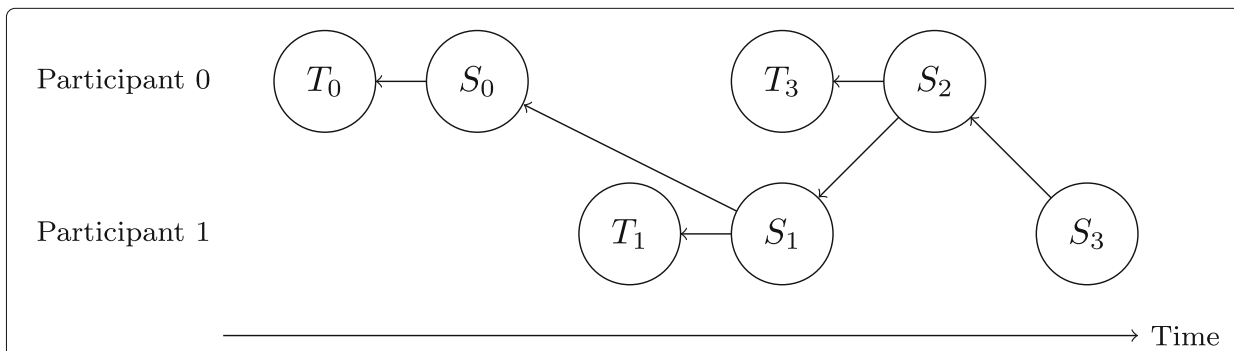

Fig. 4 Exemplified run of the optimization protocol. Two participants send transactions $T_{0}$ to $T_{3}$ and propose corresponding solutions $S_{0}$ to $S_{3}$. Both participants finally agree on solution $S_{3}$ 
Solutions include the hash values of all transactions they incorporate and also a link to any previous solutions. If a solution is accepted by a participant, they sign this solution with their private key and include it in subsequent solutions.

\section{Load Curtailment Agreement}

After one period (e.g., 24 h) all participants must agree on an optimal solution given the constraints posed by the transactions for production and consumption. Due to the requirements for valid transactions, the optimal solution is the last transaction in the DAG that has been signed by the majority of the participants.

\section{Compliance Monitoring}

Depending on the accounting model (particularly if it does not amount to an even/proportional distribution of the directly consumed energy but rather on actual consumption for each household), it may be necessary to perform compliance monitoring in order to discourage exploitative or malicious behavior of participants. In such a case, once an optimal solution has been found and agreed upon, the adherence of each participant to the subsequent schedule needs to be monitored and associated with contractually agreed consequences.

\section{Evaluation}

In the following, the proposed protocol is evaluated with respect to scalability, malicious behavior, effectiveness in finding a good solution and the range of functionality.

While blockchain applications often come with limited scalability in terms of storage and throughput (Croman et al. 2016), DAG-based applications are assumed to have better scalability (Benčić and Žarko 2018). In a typical setup for the proposed protocol, 100 houses constitute the current upper limit according to a survey from the UK (Walker and Simcock 2012) and one from the Brooklyn Microgrid (Mengelkamp et al. 2018b).

Unlike blockchain-based solutions, which are predominantly based on proof of work, computational resources are primarily used to find solutions to the practical issue of optimizing consumption rather than arbitrary challenges such as finding hash values fulfilling certain requirements.

Once a consumption period is over, all participants' scores have been calculated and billing is completed, the previous transactions are no longer needed. Thus, the DAG can be reset, which limits the data storage needs.

Regarding possible malicious behavior of participants, the submission of bogus transactions needs to be addressed. This can be counteracted in two ways: First, new users may only be allowed to reserve a certain percentage of the predicted production, with their share gradually increasing as they prove their trustworthiness by matching their consumption to their transactions. Second, the previously discussed scoring system, which would make it possible to ban participants that repeatedly fail to match their actual (minimal) consumption to the previously agreed amount.

In general, applications that rely on decentralized trust are subject to attacks where a majority can change the rules. In a classical Bitcoin application, this is known as the 51\%-attack (Eyal and Sirer 2014). In the proposed DAG data structure, where no mining or Proof of Work consensus is used, rather simple Byzantine fault tolerance applies, see, e.g., Eyal et al. (2016); Mazières (2016). Similarly, in the proposed protocol, the rules for 
finding a good solution only exist as an abstract consensus among all participants. If a majority of the participants decides to change the rules, their adaptation becomes the new standard.

An important criterion for assessing the effectiveness of the proposed protocol is whether a good solution is found or not. According to the criteria stated in "Preliminaries" section, it is simple to find a good solution that meets the requirements. However, this does not necessarily need to be the best solution. All participants can commit alternate solutions to the DAG and they can use their own strategies and algorithms to find such solutions. Due to the majority voting, it is guaranteed that at least a good solution is found at the end of the period, that meets the requirements of a significant portion of the participants in the LEC.

\section{Conclusion}

In this paper, we presented a novel approach towards energy consumption optimization in local energy communities. The key contribution of this work is a trust-less decentralized protocol that allows for dynamic joins and leaves of participants and for finding an optimal solution for load curtailment. The protocol does not limit the optimization algorithm, but rather each participant can apply its own approach for load curtailment. While related work in this field focuses either on market-driven load curtailment, on the impact of social networks, demand response or the use of household batteries, in this paper we presented a formalization of shiftable loads and an approach to find the optimal solution using a directed acyclic graph. The proposed protocol is evaluated with respect to scalability, malicious behavior and effectiveness. Future work focuses on an implementation and and a practical setup in a testbed environment as well as on a scoring system to value good behavior and adherence to agreed upon load curtailment plans. Furthermore, the revocation of transactions and the impact on proposed load curtailment solutions will be explored in greater detail.

\section{Appendix}

Formalization of shiftable loads as described in "Preliminaries" section.

\section{EV Charging}

$$
\begin{aligned}
& T=\left\{E^{\mathrm{tot}} ; t_{0}, \ldots, t_{6}\right\}, \\
& t_{0}=\{0,0 ; 18: 00, * ; *\}, \\
& t_{1}=\left\{E_{1}^{\min }, E_{1}^{\max } ; *, * ; 3\right\}, \\
& t_{2}=\left\{E_{2}^{\min }, E_{2}^{\max } ; *, * ; 1\right\}, \\
& t_{3}=\left\{E_{3}^{\min }, E_{3}^{\max } ; *, * ; 1\right\}, \\
& t_{4}=\left\{E_{4}^{\min }, E_{4}^{\max } ; *, * ; 1\right\}, \\
& t_{5}=\left\{e_{5}, e_{5} ; *, * ; 1\right\}, \\
& t_{6}=\left\{e_{6}, e_{6} ; *, 06: 00+1 ; *\right\}
\end{aligned}
$$




\section{PV Production}

$$
\begin{aligned}
T & =\left\{-E^{\mathrm{tot}} ; t_{0}, \ldots, t_{8}\right\}, \\
t_{0} & =\{0,0 ; 00: 00,08: 00 ; 32\}, \\
t_{1} & =\left\{-e_{1},-e_{1} ; 09: 00,10: 00 ; 4\right\}, \\
t_{2} & =\left\{-e_{2},-e_{2} ; 10: 00,11: 00 ; 4\right\}, \\
t_{3} & =\left\{-e_{3},-e_{3} ; 11: 00,12: 00 ; 4\right\}, \\
t_{4} & =\left\{-e_{4},-e_{4} ; 12: 00,13: 00 ; 4\right\}, \\
t_{5} & =\left\{-e_{5},-e_{5} ; 13: 00,14: 00 ; 4\right\}, \\
t_{6} & =\left\{-e_{6},-e_{6} ; 14: 00,15: 00 ; 4\right\}, \\
t_{7} & =\left\{-e_{7},-e_{7} ; 15: 00,16: 00 ; 4\right\}, \\
t_{8} & =\left\{-e_{8},-e_{8} ; 16: 00,00: 00+1 ; 32\right\}
\end{aligned}
$$

\section{About this supplement}

This article has been published as part of Energy Informatics Volume 2 Supplement 1, 2019: Proceedings of the 8th DACH+ Conference on Energy Informatics. The full contents of the supplement are available online at https:// energyinformatics.springeropen.com/articles/supplements/volume-2-supplement-1

\section{Authors' contributions}

This paper was written by FK (55\%), OL (40\%) and DE (5\%). The detailed contributions are as follows: The idea of this paper was developed by FK (60\%), OL (35\%) and DE (5\%). The Abstract was written by FK (100\%), the "Related Work" section was written by OL (60\%) and FK (40\%), the Preliminaries section was written by FK (60\%) and OL (40\%). The "Optimization Protocol" section was written by FK (60\%) and OL (40\%). The "Evaluation" section was written by OL (60\%) and FK (40\%). Editorial work was done by DE (100\%). All authors read and approved the final manuscript.

\section{Funding}

Funding by the Federal State of Salzburg is gratefully acknowledged. Publication of this supplement was funded by Austrian Federal Ministry for Transport, Innovation and Technology.

\section{Availability of data and materials}

Not applicable

\section{Competing interests}

The authors declare that they have no competing interests.

\section{Published: 23 September 2019}

\section{References}

Babrowski S, Heinrichs H, Jochem P, Fichtner W (2014) Load shift potential of electric vehicles in Europe. J Power Sources 255:283-293

Benčić FM, Žarko IP (2018) Distributed Ledger Technology: Blockchain Compared to Directed Acyclic Graph. Proc - Int Confer Distrib Comput Syst 2018-July:1569-1570

Cao Y, Tang S, Li C, Zhang P, Tan Y, Zhang Z, Li J (2012) An optimized EV charging model considering TOU price and SOC curve. IEEE Trans Smart Grid 3(1):388-393

Caron S, Kesidis G (2010) Incentive-Based Energy Consumption Scheduling Algorithms for the Smart Grid. In: 2010 First IEEE International Conference on Smart Grid Communications (SmartGridComm). IEEE. pp 391-396

Chetto M (2014) Optimal scheduling for real-time jobs in energy harvesting computing systems. IEEE Trans Emerg Top Comput 2(2):122-133

Croman K, Decker C, Eyal I, Gencer AE, Juels A, Kosba A, Miller A, Saxena P, Shi E, Gün Sirer E, Song D, Wattenhofer R (2016) On Scaling Decentralized Blockchains. In: International Conference on Financial Cryptography and Data Security. Springer, Christ Church, Barbados. pp 106-125

Erol-Kantarci M, Mouftah HT (2010) Wireless Sensor Networks for domestic energy management in smart grids. In: 25th Biennial Symposium on Communications. IEEE, Kingston, Canada. pp 63-66

Eyal I, Sirer EG (2014) Majority is not Enough: Bitcoin Mining is Vulnerable. In: Financial Cryptography and Data Security. ACM, Christ Church. pp 436-454

Eyal I, Gencer AE, Sirer EG, van Renesse R (2016) Bitcoin-NG: A Scalable Blockchain Protocol. In: Proceedings of the 13th Usenix Conference on Networked Systems Design and Implementation. NSDI'16. USENIX Association, Santa Clara, CA. pp 45-59

Huang Y, Warnier M, Brazier F, Miorandi D (2015) Social networking for Smart Grid users. In: IEEE 12th International Conference on Networking, Sensing and Control. ICNSC 2015. IEEE, Taipai, Taiwan. pp 438-443

Johnson D, Menezes A, Vanstone S (2001) The Elliptic Curve Digital Signature Algorithm (ECDSA). Int J Inf Secur 1(1):36-63

Karaarslan E, Adiguzel E (2018) Blockchain Based DNS and PKI Solutions. IEEE Commun Stand Mag 2(3):52-57 
Karlsson K, Jiang W, Wicker S, Adams D, Ma E, Van Renesse R, Weatherspoon H (2018) Vegvisir: A partition-tolerant blockchain for the internet-of-things. In: International Conference on Distributed Computing Systems. IEEE. pp 1150-1158

Khan RH, Khan JY (2013) A comprehensive review of the application characteristics and traffic requirements of a smart grid communications network. Comput Netw 57(3):825-845

Koirala BP, Koliou E, Friege J, Hakvoort RA, Herder PM (2016) Energetic communities for community energy: A review of key issues and trends shaping integrated community energy systems. Renew Sust Energ Rev 56:722-744

Lei P, Ma J, Jin P, Lv H, Shen L (2012) Structural design of a universal and efficient demand-side management system for Smart Grid. In: Proceedings - Power Engineering and Automation Conference, PEAM 2012. IEEE, Wuhan, China. pp 1-6

Martins R, Musilek P, Hesse HC (2016) Optimization of photovoltaic power self-consumption using linear programming. In: EEEIC 2016 - International Conference on Environment and Electrical Engineering. IEEE, Florence, Italy. pp 1-5

Mazières D (2016) The Stellar Consensus Protocol: A Federated Model for Internet-level Consensus. Tech Rep. https:// www.stellar.org/papers/stellar-consensus-protocol.pdf. Accessed 12 Apr 2019

Mengelkamp E, Notheisen B, Beer C, Dauer D, Weinhardt C (2018a) A blockchain-based smart grid: towards sustainable local energy markets. Comput Sci - Res Develop 33(1):207-214

Mengelkamp E, Gärttner J, Rock K, Kessler S, Orsini L, Weinhardt C (2018b) Designing microgrid energy markets: A case study: The Brooklyn Microgrid. Appl Energy 210:870-880

Munsing E, Mather J, Moura S (2017) Blockchains for decentralized optimization of energy resources in microgrid networks. In: 2017 IEEE Conference on Control Technology and Applications (CCTA). IEEE, Mauna Lani, HI, USA. pp 2164-2171

Nakamoto S (2008) Bitcoin: A Peer-to-Peer Electronic Cash System. Technical report. https://bitcoin.org/bitcoin.pdf. Accessed 12 Apr 2019

Palensky P, Dietrich D (2011) Demand Side Management: Demand Response, Intelligent Energy Systems, and Smart Loads. IEEE Trans Ind Informa 7(3):381-388

Rotering N, llic M (2011) Optimal charge control of plug-in hybrid electric vehicles in deregulated electricity markets. IEEE Trans Power Syst 26(3):1021-1029

Skopik F (2014) The social smart grid: Dealing with constrained energy resources through social coordination. J Syst Softw 89(1):3-18

Sorin E, Bobo L, Pinson P (2019) Consensus-Based Approach to Peer-to-Peer Electricity Markets with Product Differentiation. IEEE Trans Power Syst 34(2):994-1004

Taneja J, Culler D (2010) Towards Cooperative Grids: Sensor/Actuator Networks for Promoting Renewables. In: First IEEE International Conference on Smart Grid Communications. IEEE, Gaithersburg, MD, USA. pp 531-536

Tschorsch F, Scheuermann B (2016) Bitcoin and Beyond: A Technical Survey on Decentralized Digital Currencies. IEEE Commun Surv Tutor 18(3):2084-2123

Van der Kam MJ, Van Sark WGJHM (2014) Increasing self-consumption of photovoltaic electricity by storing energy in electric vehicle using smart grid technology in the residential sector. In: Proceedings of the 3rd International Conference on Smart Grids and Green IT Systems. SciTePress, Barcelona. pp 14-20

Van Der Schoor T, Scholtens B (2015) Power to the people: Local community initiatives and the transition to sustainable energy. Renew Sust Energ Rev 43:666-675

Walker G, Simcock N (2012) Community Energy Systems, Vol. 1. Elsevier Ltd, Amsterdam

Weniger J, Bergner J, Quaschning V (2014) Integration of PV power and load forecasts into the operation of residential PV battery systems. In: Proceedings of the 4th Solar Integration Workshop, Berlin, Germany. pp 383-390

Zakeri B, Syri S (2015) Electrical energy storage systems: A comparative life cycle cost analysis. Renew Sust Energ Rev 42(August):569-596

Publisher's Note

Springer Nature remains neutral with regard to jurisdictional claims in published maps and institutional affiliations.

\section{Submit your manuscript to a SpringerOpen ${ }^{\circ}$ journal and benefit from:}

- Convenient online submission

- Rigorous peer review

- Immediate publication on acceptance

- Open access: articles freely available online

- High visibility within the field

Retaining the copyright to your article

Submit your next manuscript at $>$ springeropen.com 